\title{
AVALIAÇÃO DA ASSISTÊNCIA PRÉ-NATAL: RELEVÂNCIA DOS EXAMES LABORATORIAIS
}

\author{
Evaluation of prenatal care: relevance of laboratory \\ examinations
}

Evaluación de la asistencia prenatal: relevancia de las pruebas

Artigo Original

\section{RESUMO}

Objetivo: Avaliar a assistência pré-natal de mulheres assistidas ao parto e verificar a relevância dos exames laboratoriais na qualidade do pré-natal. Métodos: Estudo de corte transversal desenvolvido em um hospital universitário de referência na cidade de Santa Cruz$\mathrm{RN}$, no período de junho a julho de 2014 , com 50 parturientes assistidas ao parto. As mulheres tinham entre 18-40 anos e possuíam baixo risco obstétrico. Os dados foram coletados a partir do cartão do pré-natal e aplicação de questionário estruturado. A caracterização da adequação do pré-natal foi elaborada com base nas diretrizes do Programa de Humanização do Pré-natal e Puerpério (PHPN) e no Manual Técnico de Pré-natal e Puerpério (MTPP). Realizou-se análise descritiva dos dados e o teste Qui-quadrado para a verificação de diferenças entre as proporções. Resultados: Observou-se que $86 \%(n=43)$ iniciaram o prénatal precocemente e obtiveram uma média de 7,3 consultas. Os procedimentos clínicos obstétricos tiveram cinco ou mais registros em 58\% $(n=29)$ da amostra. Quando avaliado o registro de exames laboratoriais, observou-se um baixo percentual de adequação na $2^{\mathrm{a}}$ rotina de exame preconizada (32\%, n=16). De acordo com os parâmetros (PHPN e MTPP), o prénatal esteve adequado apenas em $24 \%(n=12)$ dos casos. Quando avaliada a classificação da assistência do pré-natal sem o uso dos exames laboratoriais, a adequação subiu para 48\% $(n=24)$, com diferença estatisticamente significantes $(p<0,001)$. Conclusão: $O$ acesso ao prénatal foi satisfatório, entretanto, a qualidade do pré-natal foi deficiente e a maior fragilidade parece estar relacionada aos registros dos exames laboratoriais.

Descritores: Cuidado Pré-natal; Qualidade da Assistência à Saúde; Exames de Sangue.

\section{ABSTRACT}

Objective: To evaluate the prenatal care assistance provided to women assisted at delivery and examine the relevance of laboratory tests on the quality of prenatal care. Methods: $A$ cross-sectional cohort study carried out in a university referral hospital, in the city of Santa Cruz-RN, from June to July 2014, including 50 pregnant women assisted at delivery. The women were between 18-40 years old and presented low obstetric risk. Data was collected from the prenatal medical chart and through a structured questionnaire. The characterization of the prenatal assistance adequacy was developed based on guidelines of the Prenatal and Puerperium Humanization Program (PPHP) and the Prenatal and Postpartum Technical Manual (PPTM). A descriptive analysis of the data was performed and the chi-square test was used for verification of differences between proportions. Results: It was observed that $86 \%$ $(n=43)$ of the women initiated the prenatal care assistance early and had an average of 7.3 appointments. The clinical obstetric procedures had five or more records in $58 \%(n=29)$ of the sample. When assessed the laboratory tests records, a low percentage of adequacy was found in the 2 nd recommended routine testing $(32 \%, n=16)$. According to the parameters (PPHP and PPTM), prenatal care was suitable only in $24 \%(n=12)$ of the cases. When evaluated the classification of prenatal care assistance without the use of laboratory tests, the adequacy rose to $48 \%(n=24)$, presenting a statistically significant difference $(p<0.001)$. Conclusion: Access to prenatal care assistance was satisfactory; however, its quality was deficient, and the major weakness seems to be related to the records of laboratory tests.

Descriptors: Prenatal Care; Quality of Health Care; Blood Tests.

Recebido em: 14/08/2015 Revisado em: 09/09/2015 Aceito em: 12/12/2015

1) Universidade Federal da Paraíba - UFPB - João Pessoa (PB) - Brasil

2) Universidade Federal do Rio Grande do Norte - UFRN - Santa Cruz (RN) - Brasil

3) Universidade Federal do Rio Grande do Norte - UFRN- Natal (RN) - Brasil 


\section{RESUMEN}

Objetivo: Evaluar la asistencia prenatal de mujeres asistidas en el parto y verificar la relevancia de las pruebas de laboratorio para la calidad del prenatal. Métodos: Estudio transversal desarrollado en un hospital universitario de referencia de la ciudad de Santa CruzRN en el período entre junio y julio de 2014 con 50 parturientas asistidas en el parto. Las mujeres tenían entre 18-40 años y riesgo obstétrico bajo. Fueron recogidos los datos de la tarjeta del prenatal y aplicación de un cuestionario estructurado. La caracterización de la adecuación del prenatal fue elaborada basado en las directrices del Programa de Humanización del Prenatal y Puerperio (PHPN) y en el Manual Técnico del Prenatal y Puerperio (MTPP). Se realizó un análisis descriptivo de los datos y el test Chi-cuadrado para verificar las diferencias de las proporciones. Resultados: Se observó que el $86 \%(n=43)$ iniciaron el prenatal precoz y tuvieron una media de 7,3 consultas. Los procedimientos clínicos de la obstetricia tuvieron cinco o más registros en el 58\% $(n=29)$ de la muestra. En la evaluación del registro de las pruebas de laboratorio se observó un porcentual de adecuación bajo en la $2^{\mathrm{a}}$ rutina de prueba establecida $(32 \%, n=16)$. Según los parámetros (PHPN y MTPP), el prenatal fue adecuado solamente para el $24 \%(n=12)$ de los casos. En la evaluación de la clasificación de la asistencia del prenatal sin el uso de pruebas de laboratorio, la adecuación subió al 48\% (n=24) con diferencia estadística significativa $(p<0,001)$. Conclusión: El acceso al prenatal fue satisfactorio, sin embargo, la calidad del prenatal fue deficiente y la mayor fragilidad parece relacionarse con los registros de las pruebas de laboratorio.

Descriptores: Atención Prenatal; Calidad de la Atención de Salud; Pruebas Hematológicas

\section{INTRODUÇÃO}

A redução da mortalidade materno-infantil permanece como um dos focos na saúde pública e constitui uma das metas do milênio ${ }^{(1,2)}$. No Brasil, a maioria das mortes e complicações durante a gravidez, o parto e o puerpério tem como principais causas as síndromes hipertensivas, hemorragias e infecções, as quais são passíveis de prevenção através de uma adequada assistência durante o período prénatal ${ }^{(3-5)}$.

A assistência pré-natal de qualidade contribui para desfechos mais favoráveis e permite a detecção e o tratamento oportuno de afecções, além de controlar possíveis complicações para a saúde da mulher e do bebê, auxiliando na redução da morbimortalidade materna e neonatal ${ }^{(6)}$.

Nesse sentido, a Organização Mundial da Saúde estabeleceu (OMS) estabeleceu um modelo, elaborado a partir da revisão sistemática de ensaios clínicos randomizados de vários países, que preconiza as ações a serem desenvolvidas, a fim de otimizar os recursos sem comprometer a saúde das gestantes de baixo risco gestacional $^{(7)}$. No Brasil, o Ministério da Saúde estabeleceu o modelo de atendimento pré-natal através do Programa de Humanização no Pré-natal e Nascimento (PHPN), o qual normatiza as medidas a serem adotadas durante o pré-natal e puerpério, para garantir o acesso e a melhoria da cobertura e da qualidade do acompanhamento prénatal no modelo de atenção integral, assistência ao parto, puerpério e assistência neonatal ${ }^{(8)}$. A avaliação dos critérios estabelecidos pelo PHPN durante o processo da assistência pré-natal tem sido realizada em vários estudos, visando identificar o cumprimento e o desempenho do serviço e avaliar a qualidade da assistência ${ }^{(6,9,10)}$.

Com intuito de reforçar as medidas propostas pelo PHPN e organizar a rede assistencial e a normatização das práticas de saúde, foi lançado o Manual Técnico Pré-natal e Puerpério: atenção qualificada e humanizada (MTPP), que trouxe os procedimentos recomendados durante todo o acompanhamento pré-natal, incluindo os parâmetros clínicos obstétricos ${ }^{(11)}$. Além disso, em 2011, o Brasil lançou a Rede Cegonha, uma estratégia instituída pelo Ministério da Saúde que visa implementar uma rede de cuidados para assegurar às mulheres uma melhor qualidade da assistência durante o pré-natal, parto e puerpério ${ }^{(3)}$.

As pesquisas que visam avaliar a assistência durante o pré-natal constituem-se uma potente ferramenta norteadora para o conhecimento dos avanços e fragilidades ainda existentes nas redes de atenção à saúde, contribuindo para gerar subsídios aos gestores de saúde no planejamento e na implementação de medidas para redução da morbimortalidade materna e neonatal. Dessa forma, este estudo teve como objetivo avaliar a assistência pré-natal de mulheres assistidas ao parto e verificar a relevância dos exames laboratoriais na qualidade do pré-natal.

\section{MÉTODOS}

Trata-se de um estudo transversal, desenvolvido no Hospital Universitário Ana Bezerra (HUAB), localizado no município de Santa Cruz-RN. O HUAB é considerado uma unidade referência na microrregião da Borborema Potiguar, no tocante ao atendimento especializado à saúde da gestante e da criança.

O estudo ocorreu no período de junho a julho de 2014, com 50 parturientes advindas da microrregião da Borborema Potiguar, todas assistidas ao parto no HUAB. Foram consideradas elegíveis as de baixo risco obstétrico (sendo essa informação observada no prontuário e no cartão da gestante) e as que tinham entre 18 e 40 anos. Não entraram no estudo as parturientes que não tinham o cartão da gestante ou não realizaram nenhuma consulta de prénatal. 
Para a coleta dos dados, realizaram-se entrevistas em até 24 horas após o parto, utilizando-se um questionário estruturado padronizado, elaborado para a realização desta pesquisa e baseado na literatura. O questionário contemplou dados pessoais e sociodemográficos, abordando variáveis como: idade, etnia, estado civil, grau de escolaridade e renda familiar.

Para avaliar os dados referentes à gestação e ao prénatal, foram coletadas informações sobre paridade e tipo de parto. A fonte de dados para a avaliação da qualidade do pré-natal compreendeu os registros do cartão de prénatal, do qual foram coletadas as informações referentes aos procedimentos clínicos (inicio do pré-natal, número de consultas pré-natais, registro de operação de peso e pressão arterial) e laboratoriais (tipo sanguíneo - $\mathrm{ABO} /$ $\mathrm{Rh}$, hemoglobina e hematócrito $-\mathrm{Hb} / \mathrm{Ht}$, sorologia para sífilis - VDRL (Venereal Disease Research Laboratory), sumário de urina - EQU, glicemia de jejum e teste antiHIV) realizados durante a gestação.

O indicador para a avaliação da adequação da assistência recebida no pré-natal teve sua construção a partir da junção dos critérios instituídos pelo Programa de Humanização no Pré-natal e Nascimento (PHPN) $)^{(8)}$ e pelo Manual Técnico de Pré-natal e Puerpério (MTPP) ${ }^{(11)}$.

A classificação da assistência realizou-se em três categorias: Adequada - quando no cartão da gestante estavam registradas seis ou mais consultas de pré-natal e início do pré-natal antes de vinte semanas de gestação; cinco ou mais registros dos procedimentos de aferição da pressão arterial, do peso, do cálculo da idade gestacional e da altura uterina; quatro ou mais registros dos batimentos cardiofetais e, pelo menos, um registro na primeira consulta dos exames de: ABO/RH, Ht/Hb, VDRL, EQU, glicemia de jejum, teste anti-HIV e novo registro próximo à $30^{\mathrm{a}}$ semana de gestação dos exames de VDRL, EQU e glicemia. Inadequada - quando no cartão da gestante constavam: registro da primeira consulta do pré-natal após 28 semanas de gestação ou menos de três consultas registradas; dois ou menos registros da altura uterina, idade gestacional, pressão arterial, peso, batimentos cardiofetais e nenhum registro dos exames laboratoriais. Intermediária - quando a avaliação se enquadrou nas demais situações.

$\mathrm{Na}$ tentativa de verificar a relevância dos exames laboratoriais na adequação do pré-natal, a classificação da assistência também foi realizada retirando-se dos critérios estabelecidos os referidos exames, constituindo o indicador PHPN/MTPP modificado.

Os dados foram armazenados em software específico, com dupla entrada, para facilitar a detecção de possíveis erros de digitação e as análises estatísticas realizadas no SPSS, versão 20.0. A análise dos dados envolveu procedimentos de estatística descritiva e análises bivariadas. Utilizou-se o teste Qui-quadrado para a verificação de diferenças entre as proporções, adotando-se a significância estatística de $\mathrm{p}<0,05$.

Este estudo passou previamente pela aprovação do Comitê de Ética em Pesquisa do Hospital Universitário Onofre Lopes (HUOL), da Universidade Federal do Rio Grande do Norte (UFRN), sob o parecer de número 289.309. Todos os dados tiveram sua coleta somente após o aceite voluntário e assinatura do Termo de Consentimento Livre e Esclarecido pelas participantes.

\section{RESULTADOS}

A amostra foi composta por 50 participantes com média de idade de 25,8 $\pm 5,1$ anos, com maioria distribuída na faixa etária de 18 a 24 anos (46\%; n=23) (Tabela I).

As participantes eram predominantemente de cor parda $(64,6 \% ; n=31)$, casadas ou viviam em união consensual $(84 \% ; n=42)$, e possuíam até o ensino fundamental completo $(59,2 \% ; n=29)$. A maioria das famílias das participantes possuía renda de até um salário mínimo $(84 \% ; n=42)$ e era composta por uma média de $3,7 \pm 1,5$ pessoas por domicílio (Tabela I).

As mulheres apresentavam paridade anterior ao último parto, variando entre 0 e 2 filhos (média de $1,45 \pm 1,4$ ). Com relação à via do parto, observou-se que a maior parte $(78 \%$; $n=39$ ) teve parto normal no período da pesquisa (Tabela I).

Tratando-se da qualidade do pré-natal, de acordo com os parâmetros descritos no PHPN/MTPP, para a maioria $(70 \% ; n=35)$ das participantes, a assistência foi classificada como intermediária, estando apenas $24 \%(\mathrm{n}=12)$ dos casos em adequação.

Ao serem aplicados testes para avaliar se havia relação entre a qualidade do pré-natal e as características sociodemográficas da população, observou-se que, estatisticamente, essas variáveis eram independentes (Tabela I).

A avaliação dos critérios usados na classificação do pré-natal de forma isolada está descrita na Tabela II. Os dados demonstram o número de média de 7,3 $\pm 2,7$ consultas realizadas pelas, então, gestantes. Além disso, $86 \%(n=43)$ das entrevistadas tiveram início precoce da assistência, sendo a primeira consulta realizada antes da $20^{a}$ semana gestacional. Os registros dos procedimentos clínicos obstétricos realizados em cada consulta também estavam, em sua maioria com $58 \%(n=29)$, com a frequência recomendada ( $\geq 5$ registros), principalmente no que se refere ao peso $(80 \% ; n=40)$ e pressão arterial $(86 \%$; $\mathrm{n}=43$ ). Quanto ao registro dos batimentos cardiofetais, cuja recomendação é de $\geq 4$ registros, no presente estudo a média encontrada foi de $4,2 \pm 2,4$ registros. 
Tabela I - Características gerais das parturientes e neonatos incluídos na pesquisa, segundo classificação da qualidade do pré-natal. Santa Cruz, RN, 2014.

\begin{tabular}{|c|c|c|c|c|c|}
\hline \multirow{2}{*}{ Variáveis } & \multirow[t]{2}{*}{ n (\%) } & \multicolumn{3}{|c|}{ Adequação do pré-natal [n (\%)] } & \multirow{2}{*}{ Valor de $\mathbf{p}^{\ddagger}$} \\
\hline & & Adequado & Intermediário & Inadequado & \\
\hline \multicolumn{6}{|l|}{ Idade $(\operatorname{anos})(\mathrm{n}=\mathbf{5 0})$} \\
\hline$\leq 20$ anos & $9(18)$ & $2(22,2)$ & $6(66,7)$ & $1(11,1)$ & 0,68 \\
\hline $21-24$ & $14(28)$ & $5(35,7)$ & $8(57,1)$ & $1(7,1)$ & \\
\hline $25-30$ & $15(30)$ & $2(13,3)$ & $12(80)$ & $1(6,7)$ & \\
\hline$>30$ & $12(24)$ & $3(25)$ & $9(75)$ & 0 & \\
\hline \multicolumn{6}{|l|}{ Etnia $(n=48)$} \\
\hline Branco & $14(29,2)$ & $2(14,3)$ & $12(85,7)$ & 0 & 0,14 \\
\hline Pardo & $31(64,6)$ & $9(29,0)$ & $20(64,5)$ & $2(6,5)$ & \\
\hline Negro & $1(2,1)$ & $1(100)$ & 0 & 0 & \\
\hline Amarelo & $2(4,2)$ & 0 & $1(50)$ & $1(50)$ & \\
\hline \multicolumn{6}{|l|}{ Estado Civil (n=50) } \\
\hline Casada $^{\mathrm{a}}$ & $42(84)$ & $10(23,8)$ & $30(71,4)$ & $2(4,7)$ & 0,73 \\
\hline Não casada ${ }^{b}$ & $8(16)$ & $2(25)$ & $5(62,5)$ & $1(12,5)$ & \\
\hline \multicolumn{6}{|l|}{ Grau de escolaridade $(n=49)$} \\
\hline Alfabetizado & $7(14,3)$ & $3(42,9)$ & $4(57,1)$ & 0 & 0,20 \\
\hline Ensino fundamental completo & $22(44,9)$ & $3(13,6)$ & $17(77,3)$ & $2(9,1)$ & \\
\hline Ensino médio completo & $20(40,8)$ & $6(30)$ & $14(70)$ & 0 & \\
\hline \multicolumn{6}{|l|}{ Renda Familiar $(\mathrm{n}=50)$} \\
\hline Até 1 salário mínimo† & $42(84)$ & $9(21,4)$ & $30(71,4)$ & $3(7,1)$ & 0,40 \\
\hline De 1 a 2 salários mínimos & $6(12)$ & $3(50)$ & $3(50)$ & 0 & \\
\hline$\geq 2$ salários mínimos & $2(4)$ & 0 & $2(100)$ & 0 & \\
\hline \multicolumn{6}{|l|}{$N^{0}$ de pessoas/domicílio $(n=49)$} \\
\hline Até 2 pessoas & $10(20,4)$ & $3(30)$ & $7(70)$ & 0 & 0,51 \\
\hline De 3 a 5 pessoas & $33(67,3)$ & $9(27,3)$ & $22(66,7)$ & $2(6,1)$ & \\
\hline Mais de 5 pessoas & $6(12,3)$ & 0 & $5(83,3)$ & $1(16,7)$ & \\
\hline \multicolumn{6}{|l|}{ Ocupação (n=49) } \\
\hline Agricultura & $6(12,2)$ & $2(33,3)$ & $4(66,7)$ & 0 & 0,94 \\
\hline Indústria e comércio & $3(6,1)$ & $1(33,3)$ & $2(66,7)$ & 0 & \\
\hline Funcionária do governo e liberal & $2(4,1)$ & 0 & $2(100)$ & 0 & \\
\hline Do lar & $36(73,5)$ & $7(19,5)$ & $26(72,2)$ & $3(8,3)$ & \\
\hline Outros & $2(4,1)$ & $1(50)$ & 0 & $1(50)$ & \\
\hline \multicolumn{6}{|l|}{ Paridade $(n=49)$} \\
\hline 0 (Nulípira) & $14(28,6)$ & $3(21,4)$ & $11(78,6)$ & 0 & 0,23 \\
\hline 1 (Primípara) & $16(32,7)$ & $2(12,5)$ & $12(75)$ & $2(12,5)$ & \\
\hline 2 (Secundípara) & $10(20,4)$ & $5(50)$ & $5(50)$ & 0 & \\
\hline$\geq 3$ (Multípara) & $9(18,4)$ & $2(22,2)$ & $6(66,7)$ & $1(11,1)$ & \\
\hline \multicolumn{6}{|l|}{ Via de parto } \\
\hline Normal & $39(78)$ & $8(20,5)$ & $28(71,8)$ & $3(7,7)$ & 0,30 \\
\hline Cesárea & $11(22)$ & $4(36,4)$ & $7(63,6)$ & 0 & \\
\hline
\end{tabular}

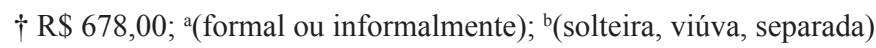

\# teste Qui-quadrado. 
Na Tabela III, são apresentados os exames laboratoriais registrados no cartão do pré-natal. O percentual de realização dos exames que devem ser realizados na primeira consulta pré-natal, conforme preconizado pelo PHPN, foi considerado satisfatório $-64 \%(n=32)$, uma vez que foram identificados elevados índices de registro de glicemia e VDRL, ambos com 91,8\% ( $\mathrm{n}=46)$; grupo sanguíneo/fator RH e hematócrito/hemoglobina, com frequência de $89,8 \%$ $(\mathrm{n}=45)$; sumário de urina, $87,8 \%(\mathrm{n}=44)$; e HIV, 73,5\% $(\mathrm{n}=37)$.

Todavia, quando avaliado o registro dos exames que devem ser repetidos próximo à $30^{\mathrm{a}}$ semana de gestação (segunda rotina de exames), observou-se uma importante redução destes $(32 \% ; n=16)$. Nesse último período, os exames de glicemia de jejum e VDRL, por exemplo, estiveram presentes somente em $36,7 \%(\mathrm{n}=18)$ e $36,6 \%$ $(\mathrm{n}=18)$ dos cartões das entrevistadas, respectivamente. Já o sumário de urina foi o exame que obteve maior número de registros, constando em 42,9\% $(n=21)$ dos cartões avaliados. Quanto ao exame de anti-HIV, o qual, segundo o PHPN, sempre que possível deve ser repetido no terceiro trimestre de gestação, constou somente em $6,1 \%(n=3)$ dos cartões analisados, quando verificados os registros da segunda rotina de exames.

Ao se verificar, a partir da utilização do PHPN/MTPP modificado (PHPN/MTPP sem exames laboratoriais), se a ausência dos exames laboratoriais estava contribuindo para a baixa qualidade do pré-natal, o percentual de adequação subiu para $48 \%(n=24)$. As mães, anteriormente classificadas

Tabela II - Realização dos procedimentos clínicos obstétricos durante o pré-natal. Santa Cruz, RN, 2014.

\begin{tabular}{|c|c|c|}
\hline Variáveis & Média ( \pm DP) & n (\%) \\
\hline Idade gestacional de início & & - \\
\hline$<20$ semanas & $12,2( \pm 6,8)$ & $43(86)$ \\
\hline $20-28$ semanas & & $4(8)$ \\
\hline$>28$ semanas & & $3(6)$ \\
\hline$N^{0}$ de consultas no pré-natal & & - \\
\hline$\geq 6$ consultas & $7,3( \pm 2,7)$ & $38(76)$ \\
\hline $3-5$ consultas & & $9(18)$ \\
\hline$<3$ consultas & & $3(6)$ \\
\hline Peso & & - \\
\hline$\geq 5$ registros & $6,8( \pm 2,4)$ & $40(80)$ \\
\hline $3-4$ registros & & $6(12)$ \\
\hline$\leq 2$ registros & & $4(8)$ \\
\hline Pressão arterial & & - \\
\hline$\geq 5$ registros & $6,9( \pm 2,3)$ & $43(86)$ \\
\hline $3-4$ registros & & $4(8)$ \\
\hline$\leq 2$ registros & & $3(6)$ \\
\hline Cálculo da idade gestacional & & - \\
\hline$\geq 5$ registros & $7,3( \pm 2,7)$ & $43(86)$ \\
\hline $3-4$ registros & & $4(8)$ \\
\hline$\leq 2$ registros & & $3(6)$ \\
\hline Altura uterina & & - \\
\hline$\geq 5$ registros & $5,2( \pm 2,5)$ & $34(68)$ \\
\hline $3-4$ registros & & $8(16)$ \\
\hline$\leq 2$ registros & & $8(16)$ \\
\hline Batimentos cardiofetais & & - \\
\hline$\geq 4$ registros & $4,2( \pm 2,4)$ & $31(62)$ \\
\hline$=3$ registros & & $6(12)$ \\
\hline$\leq 2$ registros & & $13(26)$ \\
\hline
\end{tabular}

$\mathrm{DP}=$ desvio padrão. 
como qualidade intermediária, foram consideradas com pré-natal adequado em um percentual significativo, como pode ser observado na Figura 1. Desse modo, aplicou-se o teste Qui-quadrado e observou-se diferença estatisticamente significante $(\mathrm{p}<0,001)$ entre esse percentual e a proporção anteriormente encontrada de $24 \%(n=12)$ (Figura 1).

Tabela III - Realização de exames laboratoriais preconizados durante o pré-natal. Santa Cruz, RN, 2014.

\begin{tabular}{lcc}
\hline Exames realizados & $\mathbf{1}^{\mathbf{a}}$ Rotina de exames & $\mathbf{2}^{\mathbf{a}}$ Rotina de exames \\
\cline { 2 - 3 } (n=49) & $\mathbf{n}(\mathbf{\%})$ & $\mathbf{n}(\mathbf{\%})$ \\
\hline Grupo sanguíneo e fator Rh & - & - \\
Sim & $44(89,8)$ & \\
Não & $5(10,2)$ & - \\
Hemoglobina/ Hematócrito & - & \\
Sim & $44(89,8)$ & - \\
Não & $5(10,2)$ & - \\
Teste anti-HIV & - & - \\
Sim & $36(73,5)$ & $18(36,7)$ \\
Não & $13(26,5)$ & $31(63,3)$ \\
Glicemia & - & - \\
Sim & $45(91,8)$ & $21(42,9)$ \\
Não & $4(8,2)$ & $28(57,1)$ \\
Sumário de urina & - & - \\
Sim & $43(87,8)$ & $18(36,6)$ \\
Não & $6(12,2)$ & $31(63,3)$ \\
Teste VDRL & - & \\
Sim & $45(91,8)$ & \\
Não & $4(8,2)$ & \\
\hline
\end{tabular}

HIV: Vírus da imunodeficiência adquirida; VDRL: Venereal Disease Research Laboratory

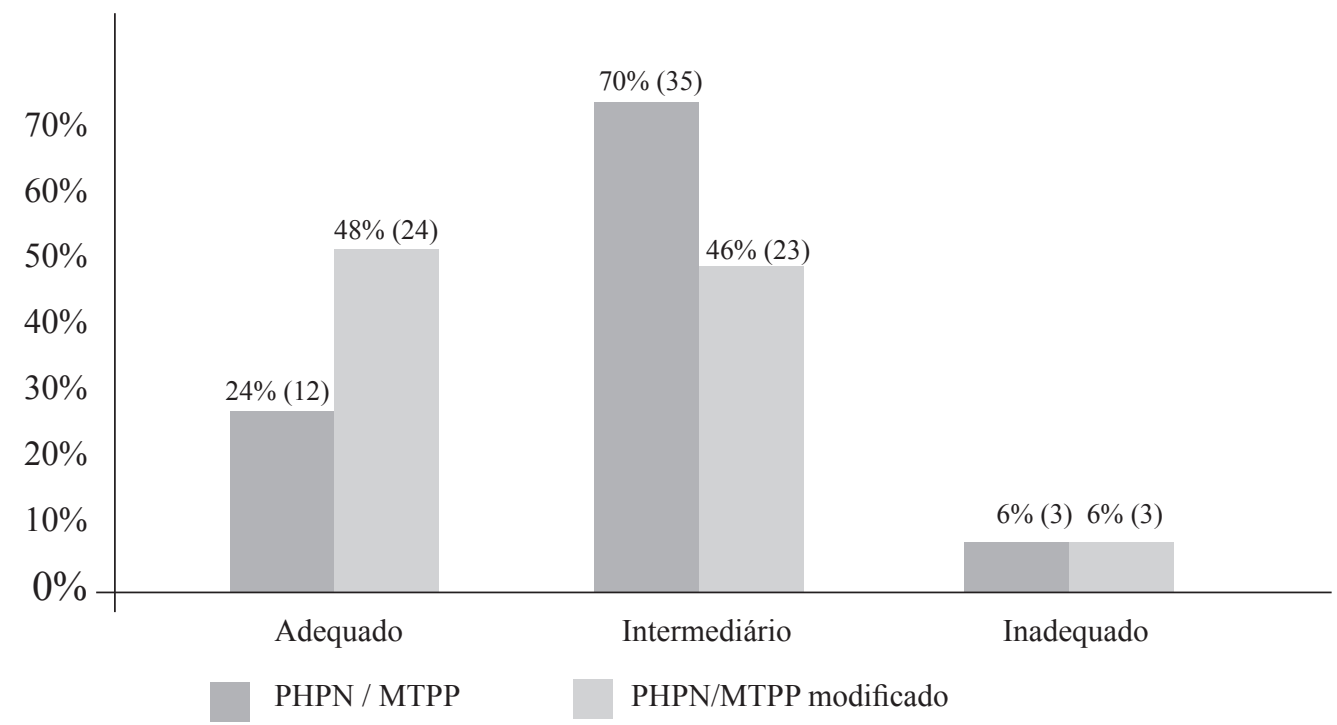

Figura 1 - Adequação pré-natal com e sem os exames laboratoriais. Santa Cruz-RN, 2014.

Dados demonstrados em percentuais e frequência; PHPN/MTPP = índice criado a partir dos critérios clinicos e laboratorias do Programa de Humanização no Pré-natal e Nascimento (PHPN)/Manual Técnico de Pré-natal e Puerpério. PHPN/MTPP modificado = índice criado a partir dos critérios clinicos sem a presença dos exames laboratoriais de acordo com Programa de Humanização no Pré-natal e Nascimento (PHPN)/Manual Técnico de Pré-natal e Puerpério. 


\section{DISCUSSÃO}

Os dados obtidos no presente estudo identificaram elevada cobertura da assistência de acordo com os critérios preconizados pelo $\mathrm{PHPN}^{(8)} \mathrm{e} \mathrm{MTPP}^{(11)}$, todavia, observou-se uma baixa frequência de mulheres com a assistência prénatal classificada como adequada, destacando essa baixa qualidade quando incluída nos critérios a realização dos exames laboratoriais.

O Ministério da Saúde enfatiza que o cuidado na atenção pré-natal deve ser integral e acontecer desde o início da gestação, visando o acompanhamento e melhores desfechos quanto à saúde da mãe e do feto ${ }^{(6)}$, uma vez que o pré-natal com qualidade é tido como o principal alvo a ser atingido quando se busca reduzir as taxas de morbimortalidade materna e infantil ${ }^{(6,12)}$.

Diante disso, a qualidade do pré-natal passou a ser avaliada por meio do número de consultas e da idade gestacional de ingresso no serviço de saúde ${ }^{(4,12)}$. Nesse contexto, o início precoce de acompanhamento e o número de consultas realizadas durante todo o período pré-natal, encontrados na presente pesquisa, foram considerados favoráveis e se assemelham ao que tem sido observado em outros estudos ${ }^{(9,10,12)}$ A importância desses achados se dá pelo fato de que uma maior cobertura da assistência fornecida à gestante pode significar mais oportunidade dela receber cuidados de prevenção e promoção à saúde. No entanto, a literatura também demonstra que apenas esses parâmetros não são suficientes para garantir uma assistência de qualidade, devendo também se avaliar o conteúdo do atendimento oferecido a essas gestantes ${ }^{(10,13,14)}$. Assim, o acompanhamento durante o pré-natal deve priorizar uma atenção qualificada e humanizada, e promover ações que integrem todos os níveis de atenção da assistência, atendendo às diretrizes do SUS e aos princípios instituídos pelo $\mathrm{PHPN}^{(8)}$, a partir dos quais são estabelecidos critérios para uma atenção materna voltada não apenas para o número de consultas e a idade gestacional de ingresso, mas também aos aspectos assistenciais e clínicos, e ações de educação em saúde, que complementam a avaliação do pré-natal na atualidade $^{(8,10,14,15)}$.

Pesquisa recente realizada com 23.894 mulheres em âmbito hospitalar, que utilizaram como critérios para a classificação da qualidade da assistência prénatal as características clínicas obstétricas e exames laboratoriais, semelhantes aos adotados no presente estudo, apresentou frequência de adequação do pré-natal de $24 \%$, corroborando com os dados encontrados neste estudo ${ }^{(10)}$. Em contraponto, outro estudo, que avaliou a qualidade do pré-natal empregando outros índices de classificação, baseados unicamente no número de consultas e/ou início do pré-natal, encontrou percentuais superiores de pré-natais adequados $^{(12)}$. Esses achados demonstram a importância de se avaliar diversos parâmetros utilizados no acompanhamento pré-natal, como realizado no atual estudo.

A presente pesquisa, ao analisar os procedimentos clínicos realizados durante o pré-natal, tendo como referência o $\mathrm{MTPP}^{(11)}$, verificou elevada frequência da aferição de todos os parâmetros, entre eles o peso, o cálculo da idade gestacional, a aferição da pressão arterial e da altura uterina. Esses resultados estão de acordo com outros estudos, segundo os quais a verificação do peso da gestante e a aferição da pressão arterial foram os procedimentos com menor percentual de ausência ${ }^{(13,14,16)}$.

Quanto à realização dos exames laboratoriais durante a gestação, ela é considerada oportuna para prevenir, identificar e corrigir as anormalidades que possam afetar a gestante e seu concepto, assim como instituir o tratamento de doenças já existentes ou que possam ocorrer durante a gestação ${ }^{(4)}$. No Nordeste do Brasil, em $89,1 \%$ casos de nascidos vivos, as mães fizeram pelo menos um exame durante a gestação ${ }^{(7)}$. Dados que corroboram com o presente estudo, no qual se observou que o registro de hemograma/ hematócrito também apresentou relevante frequência nos cartões, quando verificada a presença deste exame na primeira consulta gestacional. $\mathrm{O}$ mesmo aconteceu para outros exames laboratoriais preconizados pelo PHPN, como glicemia, tipagem sanguínea, HIV e sorologia para sífilis.

Entretanto, um dado preocupante encontrado no presente estudo ocorreu ao se observarem as informações de exames recomendados próximos à $30^{\mathrm{a}}$ semana de gestação (segunda rotina), quando houve uma diminuição importante no número de registros dos exames laboratoriais, sendo os testes de HIV e VDRL os exames que apresentaram a maior redução. Estudo anterior realizado com gestantes observou resultados semelhantes aos da presente pesquisa, em que metade da população de gestantes avaliadas realizou a sorologia anti-HIV durante o pré-natal apenas uma vez e pouco mais de um quarto da amostra realizou esse exame duas vezes ${ }^{(15)}$.

Diante desses fatos, são preocupantes os altos percentuais de falta de registro de variáveis relacionadas a intercorrências maternas importantes, como a anemia, as infecções do trato urinário, o diabete mellitus, a sífilis e o HIV positivo ${ }^{(16,17)}$. A transmissão vertical do HIV e da sífilis, por exemplo, é um desafio na saúde pública que necessita ser enfrentado pelas políticas de saúde do Brasil, apesar dos avanços já obtidos nesta área. Mesmo não sendo considerada no presente estudo a presença do teste antiHIV na segunda rotina de exames para a adequação do prénatal, a execução desse exame, sempre que possível, como recomendado pelo PHPN, é de suma importância, uma vez que a taxa de transmissão do HIV da mãe para o filho, 
sem qualquer tratamento, pode ser de $20 \%$, e cerca de $65 \%$ desses casos podem ocorrer no periparto ${ }^{(15,18)}$.

A incidência da sífilis congênita também representa um importante indicador da qualidade da atenção maternoinfantil, pois sua forma congênita é uma importante causa de mortalidade perinatal ${ }^{(10,18)}$. Sua prevenção é realizada unicamente durante o pré-natal, não podendo ser feita no interparto ou pós-natal, ressaltando a relação direta entre a frequência da enfermidade e a qualidade dos serviços da rede de atenção básica prestados à mulher ${ }^{(19,20)}$. No presente estudo, observou-se uma diminuição significativa da realização do exame para detecção dessa patologia, o VDRL após a $30^{\mathrm{a}}$ semana, sendo um fator preocupante, tendo em vista a gravidade dessa doença e suas repercussões na saúde materno-infantil.

$\mathrm{Na}$ presente pesquisa, o não registro/realização dos exames bioquímicos preconizados pelo PHPN foi considerado um dos principais pontos críticos para assegurar a qualidade da assistência pré-natal. Isso foi verificado após a retirada intencional dos exames laboratoriais dos critérios da qualidade do pré-natal, comprovando a criticidade da realização e, principalmente, da repetição desses exames. Dados semelhantes foram descritos em estudo recente realizado no Espírito Santo, no qual mais de mais de 20\% das gestantes não realizaram sorologia para sífilis e um número ainda maior, cerca de $30 \%$, não realizou o exame anti-HIV. Esses dados demonstram que a não repetição desses exames tão importantes, como VRDL e anti-HIV, representa uma falha que impacta diretamente na prevenção e tratamento precoces, comprometendo o controle da transmissão vertical do HIV e a erradicação da sífilis congênita no país ${ }^{(9)}$.

Contudo, no atual estudo, uma vez que a coleta de dados foi realizada a partir do cartão da gestante, não foi possível identificar a causa da ausência dessas informações, sendo uma limitação do presente estudo. A falta desse tipo de informação pode refletir o não registro dos resultados no cartão da gestante pelos profissionais de saúde, a não solicitação desses exames, ou, ainda, a dificuldade na realização ou na obtenção dos resultados por falhas na execução dos exames, falta de insumos específicos, equipamentos danificados, extravio das amostras, demora no retorno dos resultados, entre outros motivos, ocasionando o desperdício de recursos, como visto em alguns estudos ${ }^{(9,16,21,22)}$. Tal fato torna-se preocupante, visto que as gestantes podem terminar o pré-natal sem nenhum registro em seus prontuários ${ }^{(21,23)}$.

Outra limitação encontrada no atual estudo foi a pequena casuística estudada, o que torna necessária a continuação de pesquisas envolvendo um maior número de casos e a inclusão de mulheres com gestações consideradas de risco.
Diante dos fatos apresentados, os dados do presente estudo verificam que, embora o acesso ao pré-natal tenha iniciado, em sua maioria precocemente e com o número de consultas adequadas, a qualidade dessa atenção ainda não é a ideal, uma vez que somente esses parâmetros não garantem assistência de qualidade, pois esta dependerá da realização do conjunto de ações consideradas nos critérios mínimos exigidos no $\mathrm{PHPN}^{(8)}$ e $\mathrm{MTPP}^{(11)}$. Diante dessa situação, evidencia-se a necessidade de uma discussão envolvendo profissionais de saúde, gestores e comunidade, na tentativa de conscientizar a todos sobre seus direitos e deveres em relação à qualidade do pré-natal e, consequentemente, à saúde materno-infantil.

\section{CONCLUSÃO}

A assistência pré-natal neste estudo foi satisfatória, mas ainda deficiente em qualidade, tendo como maior fragilidade a falha no registro dos exames laboratoriais, os quais teriam potencial de reverter os indicadores perinatais desfavoráveis ainda observados. Estratégias direcionadas a identificar as falhas na assistência pré-natal e medidas que venham a melhorar a qualidade desta atenção são essenciais, principalmente no que concerne à realização dos exames laboratoriais, visando à diminuição dos agravos de saúde e da mortalidade materno-infantil.

\section{AGRADECIMENTOS}

Ao Hospital Universitário Ana Bezerra, por permitir a coleta de dados, e às mulheres voluntárias que aceitaram participar deste estudo.

\section{REFERÊNCIAS}

1. Souza JP. Mortalidade materna no Brasil: a necessidade de fortalecer os sistemas de saúde. Rev Bras Ginecol Obstet. 2011;33(10):273-9.

2. United Nations. The Millenium Development Goals Report.United Nations Department of Economic and Social Affairs (DESA) [serial online] 2010 [accessed on 2013 Dec 10]. Available from: http://www.un.org/ millenniumgoals/pdf/

3. Ministério da Saúde (BR), Departamento de Ações Programáticas Estratégicas. Gestação de alto risco: manual técnico. Brasília: Ministério da Saúde; 2012.

4. Anversa ETR, Bastos GAN, Nunes LN, Pizzol TSD. Qualidade do processo da assistência pré-natal: unidades básicas de saúde e unidades de Estratégia Saúde da Família em município no Sul do Brasil. Cad Saúde Pública. 2012;28(4):789-800 
5. Dias-da-Costa JS, Cesar JA, Haag CB, Watte G, Vicenzi K, Schaefer R. Inadequação do pré-natal em áreas pobres no Nordeste do Brasil: prevalência e alguns fatores associados. Rev Bras Saúde Matern Infant. 2013;13(2):101-9.

6 .Domingues MSM, Hartz ZMA, Dias MAB, Leal MC. Avaliação da adequação da assistência pré-natal na rede SUS do município do Rio de Janeiro, Brasil. Cad Saúde Pública. 2012;28(3):425-37.

7. Villar J, Ba'aqeel H, Piaggio G, Lumbiganon P, Miguel Belizán J, Farnot U, et al. WHO Antenatal Care Trial Research Group. Who Antenatal Care Randomised Trial For The Evaluation of A New Model Of Routine Antenatal Care. Lancet. 2001;357(9268):1551-64

8. Ministério da Saúde (BR). Pesquisa Nacional de Demografia e Saúde da Criança e da Mulher - PNDS 2006: dimensões do processo reprodutivo e da saúde da criança. Brasília: Ministério da Saúde; 2009.

9. Polgliane RB, Leal MC, Amorim MH, Zandonade E, Santos Neto ET. Adequação do processo de assistência pré-natal segundo critérios do Programa de Humanização do Pré-natal e Nascimento e da Organização Mundial de Saúde. Ciênc Saúde Coletiva. 2014;19(7):1999-2010.

10. Domingues RMSM, Viellas EF, Dias MAB, Torres JA, Theme-Filha MM, Gama SGN, et al. Adequação da assistência pré-natal segundo as características maternas no Brasil. Rev Panam Salud Publica. 2015;37(3):140-7.

11. Ministério da Saúde (BR). Pré-natal e puerpério: atenção qualificada e humanizada. Brasília: Ministério da Saúde; 2005.

12. Pedraza DF, Rocha ACD, Cardoso MVML. Assistência pré-natal e peso ao nascer: uma análise no contexto de unidades básicas de saúde. Rev Bras Ginecol Obstet. 2013;35(8):49-56.

13. Eudênia OB, Lima LHO, Oliveira EKB. Prenatal care in the municipality of Quixadá: a descriptive study. Online Braz J Nurs (Online). 2012;11(2):319-30.

14. Silva EP, Lima RT, Ferreira NLS, Costa MJC. Pré-natal na atenção primaria do município de João pessoa-PB: caracterização de serviços e usuárias. Rev Bras Saúde Matern Infant. 2013;13(1):29-37.

15. Quadros LCM, Meincke MK, Lopes CV, Vargas NRC, Schneider CC. Avaliando a realização de exames laboratoriais pelas gestantes durante o pré-natal. Rev Enferm Saúde. 2011;1(1):99-106.
16. Silva MB, Monteiro PS. Adequação do pré-natal em gestantes atendidas na Estratégia de Saúde da Família em Palmas-TO. Com Ciências Saúde. 2010;21(1):2130.

17. Rocha RS, Silva MGC. Assistência Pré-Natal na rede básica de Fortaleza-Ce: uma avaliação da estrutura, do processo e do resultado. Rev Bras Promoç Saúde. 2012;25(3):344-55.

18. Portal Brasil. Saúde amplia acesso a exames do prénatal e testes rápidos de gravidez [acesso em 2013 Set 5]. Disponível em: http://www.brasil.gov.br/ saude/2013/09/oferta-de-pre-natal-e-testes-rapidos-degravidez-sera-ampliada/@@nitf_galleria

19. Holanda MTCG, Barreto MA, Machado KMM, Pereira RC. Perfil epidemiológico da sífilis congênita no Município do Natal, Rio Grande do Norte - 2004 a 2007. Epidemiol Serv Saúde. 2011;20(2):203-12.

20. Serruya SJ, Lago TG, Cecatti JG. O panorama da atenção pré-natal no Brasil e o Programa de Humanização do Pré-Natal e Nascimento. Rev Bras Saúde Matern Infant. 2004;4(3):269-79.

21. Gonçalves CV, Cesar JA, Mendonza-Sassi RA. Qualidade e equidade na assistência à gestante: um estudo de base populacional no Sul do Brasil. Cad Saúde Pública. 2009;25(11):2507-16.

22. Ramos VM, Figueiredo EN, Succi RCM. Entraves no controle da transmissão vertical da sífilis e do HIV no sistema de atenção à saúde do município de São Paulo. Rev Bras Epidemiol. 2014;17(4):887-98.

23. Ramos Jr N, Harunari LM, Saraceni V, Veras MASM, Pontes RJS. Controle da transmissão de mãe para filho de doenças infecciosas no Brasil: o progresso em HIV/ AIDS e falha na sífilis congênita. Cad Saúde Pública. 2007;23(3):370-8.

\section{Endereço para correspondência:}

Dayanna Joyce Marques Queiroz

Universidade Federal da Paraíba

Campus I

Bairro: Cidade Universitária

CEP: 58059-900 - João Pessoa - PB - Brasil

E-mail: dayannajoyse@hotmail.com 